\title{
Insights on the (Auto)Photocatalysis of Ferritin
}

Natalie Saenz, ${ }^{\dagger}$ Manu Sánchez, ${ }^{\dagger}$ Natividad Gálvez, ${ }^{\dagger}$ Fernando Carmona, ${ }^{\ddagger}$ Paolo Arosio, and Jose M. Dominguez-Vera ${ }^{*}+$

${ }^{+}$Departamento de Química Inorgánica and Instituto de Biotecnología, Universidad de Granada, 18071 Granada, Spain

${ }^{\ddagger}$ Department of Molecular and Translational Medicine, University of Brescia, Viale Europa 11, 25123 Brescia, Italy

\section{ABSTRACT}

Traditionally, ferritin has been considered a photocatalyst capable of photo-oxidizing organic molecules and transferring electrons to external electron acceptors when irradiated by UV- visible light. We have designed new approaches to resolve the uncertainties regarding its photocatalytical mechanism. Experiments with an Fe(II) chelator, an electrochromic indicator, and recombinant ferritin proteins indicate that the excited electrons at the conduction band of the ferritin core do not cross the protein shell. Instead, irradiation causes the electrons to reduce the ferrihydrite core to produce $\mathrm{Fe}(\mathrm{II})$ ions. These $\mathrm{Fe}(\mathrm{II})$ ions exit the protein shell to reduce electron acceptors. In the absence of electron acceptors or chelators, Fe(II) re-enters ferritin.

\section{INTRODUCTION}

Living organisms have developed chemical machinery based on ferritin protein to manage the availability of vital, but potentially toxic, free iron. Ferritin has the dual function of detoxifying iron by oxidizing the $\mathrm{Fe}(\mathrm{II})$ ions in its catalytic ferroxidase centers and of storing Fe(III) in its cavity [1-7]. Stored ferritin iron is nontoxic and is available when needed by the cell.

Ferritins isolated from different organisms and tissues have been determined. The most studied ferritin traditionally used as a model of mammalian ferritins is found in horse spleen. Horse spleen ferritin (HSFt) consists of apoferritin, a spherical hollow shell of about $450 \mathrm{kDa}$ with a diameter of approximately $12 \mathrm{~nm}$, and an encapsulated Fe(III) ferrihydrite-like nanoparticle [8-10]. The apoferritin shell is composed of 24 
polypeptide subunits of two types: the $H$ (heavy) and the $L$ (light) subunits. The two subunits are closely related but have different functionalities. The $\mathrm{H}$ subunits play a key role in the rapid detoxification of Fe(II), as they contain the catalytic ferroxidase centers. The $L$ subunits lack ferroxidase centers and are associated with iron nucleation [11]. The assembly of the two subunits in the ferritin shell results in different types of channels leading to the central cavity. Eight hydrophilic channels allow the passage of water and metal cations into the protein core.

Iron from the native ferrihydrite nanoparticle may be removed by reduction $[7,12]$. The Fe(II) may subsequently diffuse through the hydrophilic channels to the external medium. The ferrihydrite-like core of ferritin is a semiconductor that has been proposed to function as a photocatalyst for redox reactions. Irradiation of ferritin with UV-visible light induces the promotion of electrons in the core from the valence to the conduction band, generating an electron-hole pair that then induces oxidation-reduction reactions [13-20].

Previous studies have shown that ferritin, in the presence of electron donors, is capable of reducing various metal cations, proteins, and dyes [13-16]. It has been proposed that illuminating ferritin with visible light catalyzes the photo-oxidation of organic molecules and the transfer of electrons to external electron acceptors. Thus, a variety of electron acceptors including $\mathrm{Cr}(\mathrm{VI}), \mathrm{Cu}(\mathrm{II}), \mathrm{Au}(\mathrm{III})$, viologen, and cytochrome $\mathrm{c}$ have been reduced using citrate or tartrate as sacrificial electron donors [13-16].

The detailed mechanism in which ferritin acts as a photocatalyst remains unclear. Direct contact between the ferritin core and the substrates to be oxidized (electron donors) or the substrates to be reduced (electron acceptors) requires the entry of the substrates through the ferritin shell into the cavity. Alternatively, electron transfer through the $2 \mathrm{~nm}$ apoferritin shell could occur. Although neither mechanism has been unequivocally confirmed, it has been assumed that electrons from donors fill the photoinduced electron hole in the ferritin core and excited electrons from the conduction band reduce the electron acceptors. Although some have proposed that these excited electrons cross the electron shell, others have argued that species small enough to enter through the ferritin channels could be reduced inside the ferritin shell. In either case, it has been assumed that the substrate reductants are electrons liberated from the ferritin core $[13,14,17-19]$. 
Assuming ferritin acts as a photocatalyst, the photocatalytic ferritin core could eventually undergo photocorrosion. Light excitation would reduce the iron core itself, forming Fe(II) and dissolving the ferrihydrite nanoparticle. In fact, previous work has shown that the irradiation of ferritin with UV-visible light induces a reductive mobilization of $\mathrm{Fe}(\mathrm{II})[21]$. The $\mathrm{Fe}(\mathrm{II})$ was detected with an adequate $\mathrm{Fe}(\mathrm{II})$ chelator, ferrozine ((3-(2- pyridyl)-5,6-diphenyl-1,2,4-triazine-p,p-disulfonic acid monosodium salt, hereafter $\mathrm{fz}$ ), through the formation of the $\left[\mathrm{Fe}^{\prime \prime}(\mathrm{fz})_{3}\right]$ complex. It has also been proposed that ferritin could overcome photocorrosion $[15,17]$. In this scheme, the core would remain in an active form. Since Fe(II) that diffuses through the hydrophilic channels would reach ferroxidase centers, be reoxidized to Fe(III), and return to be reincorporated into the core.

Clearly the most intricate details of the photocatalysis by ferritin remain uncertain. The results and conclusions of several studies are mutually inconsistent and require further investigation. To this end, we have designed new approaches to understand the nature of the reduction of the ferritin core in the presence of light. We question whether the reduction is solely a photochemical reaction.

\section{RESULTS AND DISCUSSION}

First, we assessed the photoreductive ability of horse spleen ferritin with electrochromic polyoxometalate $\left[\mathrm{P}_{2} \mathrm{Mo}^{\mathrm{VI}}{ }_{18} \mathrm{O}_{62}\right]^{6-}$ (POM hereafter). POM is reduced to a family of mixed-valence $\mathrm{Mo}^{\mathrm{V} I} \mathrm{Mo}^{\mathrm{V}}$ species with a characteristic deep blue color, which is easily detected by UV-vis spectroscopy, through the appearance of a broad band centered in the 700-800 $\mathrm{nm}$ range [22-24]. In this case, POM is ideal as an indicator because it cannot cross the apoferritin channels due to its large size. Moreover, POM is reduced little by citrate and conventional buffers and is unaffected by UV-vis irradiation.

As shown in Figure 1a, when a mixture of POM and horse spleen ferritin in the presence of citrate is irradiated with a UV-vis lamp (see Experimental Section), a broad band centered at $800 \mathrm{~nm}$ gradually develops, confirming the reduction of POM. Without irradiation, no POM reduction occurs. Likewise, by irradiating ferritin absence of citrate, POM is not reduced (Figure 1b). Clearly citrate supplies electrons to the ferritin core, although whether citrate must reach the core remains uncertain. Similar 
results were obtained with POM and ferritin in the presence of different buffers, TRIS, MOPS, and HEPES, with small variations in accordance with their influence on the photoactivity of ferritin as described by Watt et al. (Supporting Information S1) [18].

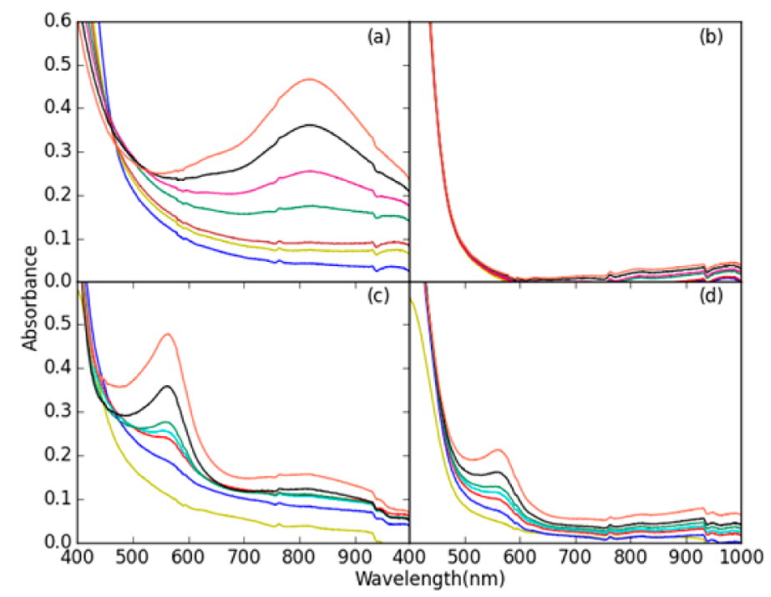

Figure 1. UV-vis spectra over time after irradiating the mixture of horse spleen ferritin (HSFt) and POM: with (a) and without citrate (b); with $f z$, with (c) and without citrate (d).

Interestingly, when $\mathrm{fz}$ is added to the mixture of POM, ferritin, and citrate and then irradiated, the typical UV-vis band of reduced POM hardly forms. Instead, a band centered at $562 \mathrm{~nm}$ appears (Figure 1c). The presence of the $562 \mathrm{~nm}$ band is indicative of the formation of the $\left[\mathrm{Fe}^{\prime \prime}(\mathrm{fz})_{3}\right]$ complex. These results indicate that after ferritin irradiation Fe(II) forms and is sequestered by $\mathrm{fz}$, hindering the reduction of POM. This preference suggests that the reduction of POM is carried out by $\mathrm{Fe}(\mathrm{II})$ and not by electrons coming from the ferritin core. In fact, in a separate experiment, Fe(II) mixed with POM and citrate resulted in the same typical UV-vis band of reduced POM (Supporting Information S2).

In light of these findings, we reviewed previously reported experiments, in which the reduction of some chemical species by irradiated ferritin was attributed to the electrons of the ferritin core [13-18]. In particular, we analyzed the reduction of $\mathrm{Au}(\mathrm{III})$ and $\mathrm{Cu}(\mathrm{II})$ to form the respective zerovalent metal nanoparticles. We have found that the reduction of these cations and the formation of gold and copper nanoparticles occurs similarly in solutions with Fe(II) as in solutions with irradiated ferritin. In both cases, the presence of citrate was necessary for the reduction. Citrate stabilizes Fe(III) 
and by lowering the $\mathrm{Fe}(\mathrm{II})-\mathrm{Fe}(\mathrm{II})$ redox potential facilitates the reduction of POM, $\mathrm{Au}(\mathrm{III})$, and $\mathrm{Cu}(\mathrm{II})$ by $\mathrm{Fe}(\mathrm{II})$.

Transmission electron microscopy (TEM) of the nanoparticles made by the addition of Fe(II) showed spherically shaped electron dense cores (Figure 2). Electron energy loss spectroscopy and energy dispersive spectroscopy confirmed that the particles contained $\mathrm{Cu}$ and $\mathrm{Au}$, according to what metal was added.

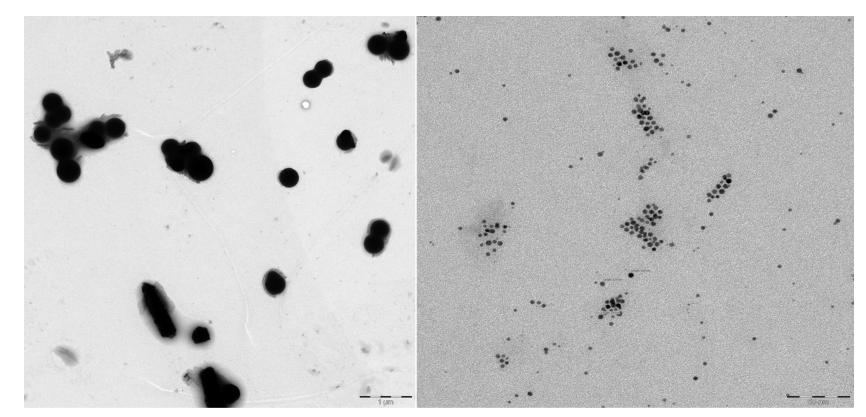

Figure 2. Typical TEM images of $\mathrm{CU}$ (left) and Au (right) nanoparticles. The scales are 1 $\mu \mathrm{m}$ and $50 \mathrm{~nm}$, respectively.

The collective analysis of these findings demonstrates that the reduction of chemical species outside ferritin does not occur through the release of electrons from the core but is due to the presence of Fe(II) leaving the ferritin shell. This mobilized Fe(II) is a product of the autophotocatalysis of ferritin in which the ferrihydrite core is reduced (Scheme 1).

\section{Scheme 1. Mechanism of Photoinduced Chemical Reduction by Ferritin. ${ }^{\text {a }}$}

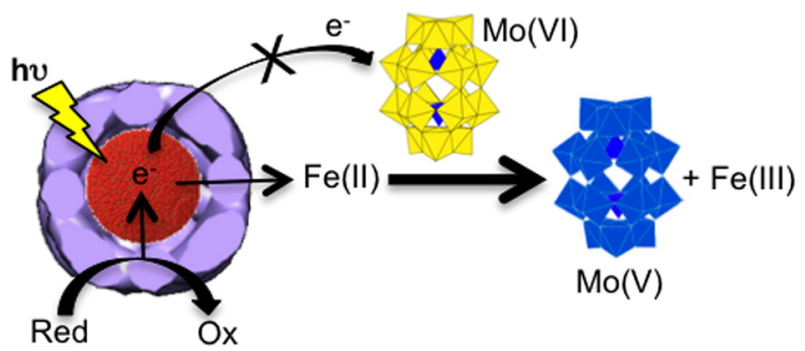

${ }^{a}$ Upon irradiation, electrons are excited from the valence band to the conduction band of the ferrihydrite semiconductor. The valence band is filled with electrons from the sacrificial electron donor. The excited electrons reduce the iron core, and Fe(II) ions are formed and liberated. 
Citrate, due to its high $\mathrm{Fe}$ (III) affinity, augments the reducing capacity of $\mathrm{Fe}$ (II). Therefore, citrate has a double role in the redox behavior of irradiated ferritin: electron donor to the iron core semiconductor and amplifier of the reducing Fe(II) reactions.

In addition, we designed experiments to address the path of Fe(II) after irradiating ferritin. It has been stipulated that $\mathrm{Fe}(\mathrm{II})$ is reoxidized at ferroxidase centers and returns to the core. If, as suggested, the ferroxidase centers stop Fe(II) from exiting, then the process of Fe(II) liberation from ferritin by irradiation should depend on the ferritin type and presence of ferroxidase centers $[15,17]$. We have addressed this issue by studying $\mathrm{Fe}(\mathrm{II})$ release by irradiation from three ferritins with equivalent iron loadings but different $\mathrm{H} / \mathrm{L}$ ratios: the recombinant human $\mathrm{H}$ - ferritin $(\mathrm{HuFtH}), a$ homopolymer of 24 identical H-subunits, each with a ferroxidase center; the horse spleen ferritin (HSFt), a heteropolymer formed by approximately three $\mathrm{H}$ - and $21 \mathrm{~L}$ subunits; and the recombinant human L-ferritin (HuFtL), a homopolymer of 24 identical L-subunits (L-subunits lack the ferroxidase center).

After irradiation, the Fe(II) liberation from HuFtH, HSFt, and HuFtL was assessed by measuring the availability of $\mathrm{Fe}(\mathrm{II})$ for $\mathrm{fz}$. The amount of iron released corresponds to the increase in UV-vis absorbance at $562 \mathrm{~nm}$, indicating the formation of the [Fe" $\left.\left(\mathrm{fz}_{3}\right)_{3}\right]$ complex. The $\mathrm{Fe}(\mathrm{II})$ availability increase patterns provide a dynamic picture of the overall iron release from ferritin induced by light. As shown in Figure 3, the patterns of iron removal are similar for the three ferritins, although HuFtH contains 24 ferroxidase centers and HuFtL none. These results support the idea that Fe(II) formed by the autophotocatalysis of ferritin is not reoxidized at a ferroxidase center but leaves the protein and is available for $\mathrm{fz}$ sequestration or for carrying out chemical reductions. 


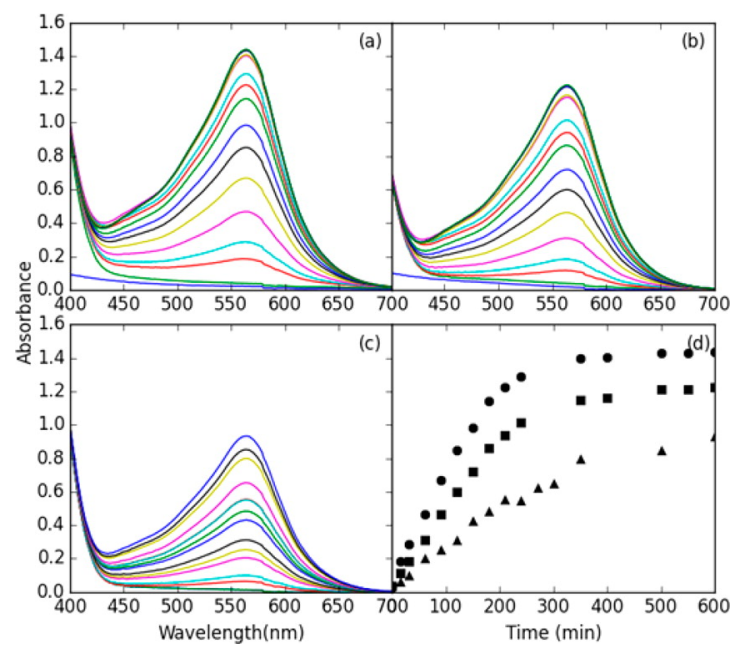

Figure 3. UV-vis spectra over time after irradiating (a) HuFtH, (b) HuFtL, and (c) HSFt in the presence of fz. (d) Absorbance at $562 \mathrm{~nm}$ over time: $\operatorname{HuFtH}(\bullet), \operatorname{HuFtL}(\mathbf{\square}), \operatorname{HSFt}(\Delta)$.

Finally, we attempted to monitor the Fe(II) release from the ferritin core in the absence of electron acceptors. Unlike the previous experiments, we added $\mathrm{fz}$ after different lapses of time of irradiation. We observed no formation of the $\left[\mathrm{Fe}^{\prime \prime}(\mathrm{fz})_{3}\right]$ complex (Figure 4). Because of the absence of $\mathrm{fz}$, the Fe(II) is not immediately captured and is available for reentry into ferritin.

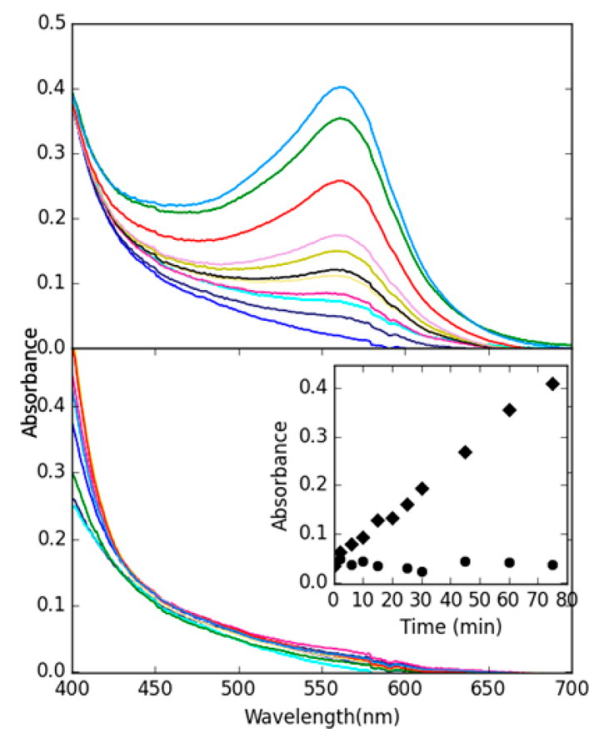

Figure 4. UV-vis spectra over time of HSFt with $f z$ added before (top) and after (bottom) irradiating. Inset is the absorbance at $562 \mathrm{~nm}$ for $\mathrm{fz}$ added from the start ( $)$ and after different times $(\bullet)$ of irradiation. 


\section{CONCLUSIONS}

Our results are inconsistent with a model in which electrons from the conduction band cross the apoferritin shell and reduce metal cations on the exterior surface of ferritin. The excited electrons instead autoreduce the ferritin core to produce $\mathrm{Fe}(\mathrm{II})$ ions. In addition, we have demonstrated that these Fe(II) ions are not reoxidized at ferroxidase centers. Rather, Fe(II) exits the shell to reduce external electron acceptors. In the absence of electron acceptors, $\mathrm{Fe}(\mathrm{II})$ is available for re-entry into ferritin.

\section{EXPERIMENTAL SECTION}

Light-Induced Ferritin Fe(II) Release with POM and Ferrozine-Fe(II) Complex Formation. Horse spleen ferritin, HSFt (approximately $2000 \mathrm{Fe} / \mathrm{Ft}$ ), was obtained from Sigma-Aldrich and was exhaustively dialyzed against Milli-Q water using a Spectra/ Por Float-A-Lyzer with a molecular weight cut-off of 300000 Da. Ferritin (25 $\mu \mathrm{M}, 12.5$ $\mathrm{mg} / \mathrm{mL}$ ) was added to solutions of $1 \mathrm{mM}$ POM with or without $30 \mathrm{mM}$ citrate. POM was obtained according to previously reported methods. 25 UV-vis spectra were taken before and after irradiating with a broad-spectrum (400-800 nm) LED lamp. Control samples were kept in darkness. Spectra were taken at 15, 30,60, and 100 min intervals as the experiment progressed. The same procedure was followed with the addition of an excess of $\mathrm{fz}$.

To compare buffers, solutions containing $1 \mathrm{mM}$ POM, $0.25 \mu \mathrm{M} \mathrm{HSFt}$, and $30 \mathrm{mM}$ of either MOPS, HEPES, or TRIS at pH 7.4 were illuminated by the broad-spectrum LED lamp and measured with UV-vis spectroscopy on the hour. After $24 \mathrm{~h}$ in the light, citrate was added to a portion of the solution, which was then measured with UV-vis spectroscopy.

Copper and Gold Nanoparticles. Copper and gold nanoparticles formed by mixing 0.1 $\mathrm{M}$ solutions of $\mathrm{FeSO} 4$ and $\mathrm{CuSO} 4$ or $\mathrm{AuCl} 3$ in 125 mM HEPES and 400 mM citrate. Electron micrographs were taken with a Phillips CM-20 HR analytical electron microscope operating at $200 \mathrm{keV}$.

Recombinant Ferritin Preparation. Recombinant human HuFtH and HuFtL were prepared as previously described.26 All ferritins were electrophoretically pure. Protein 
concentrations were determined with BCA reagent (Pierce) using bovine serum albumin as standard.

HuFtH, HuFtL, and HSFt were reconstituted in $0.1 \mathrm{M}$ imidazole/ $0.05 \mathrm{M} \mathrm{NaCl}$ pH 7.4 buffer. Fe(II) was slowly added at 20 min intervals for a total iron loading of 200 Fe/ferritin. The proteins were transferred via dialysis to $20 \mathrm{mM}$ TRIS buffer (pH 7.4, 5 $\mathrm{mM} \mathrm{NaCl})$.

Light-Induced Ferritin Fe(II) Release from HuFt. Ferritin (HuFtH, HuFtL, or HSFt) was added in $25 \mu \mathrm{M}$ amounts to solutions with $30 \mathrm{mM}$ citrate and $20 \mathrm{mM}$ TRIS. Spectra were taken before and after addition of excess $\mathrm{fz}$. Cuvettes were irradiated, and spectra were taken every 15, 30, and then 60 min intervals as the experiment progressed.

Ferrozine Control. HSFt was added in $25 \mu \mathrm{M}$ amount to a solution of $20 \mathrm{mM}$ TRIS buffer $(\mathrm{pH} 7.4,5 \mathrm{mM} \mathrm{NaCl})$. The solution was distributed among several UV-vis cuvettes. Fz was added in excess to one sample. All cuvettes were irradiated. The sample with $\mathrm{fz}$ was measured with UV-vis spectrometry at the same time as $\mathrm{fz}$ was added to another sample and measured. Spectra were taken first every 4 min and then every $15 \mathrm{~min}$.

\section{ACKNOWLEDGMENTS}

This work was funded by MINECO and FEDER (CTQ2015- 64538). N.S. would like to acknowledge funding from the U.S. Student Fulbright Scholarship. 


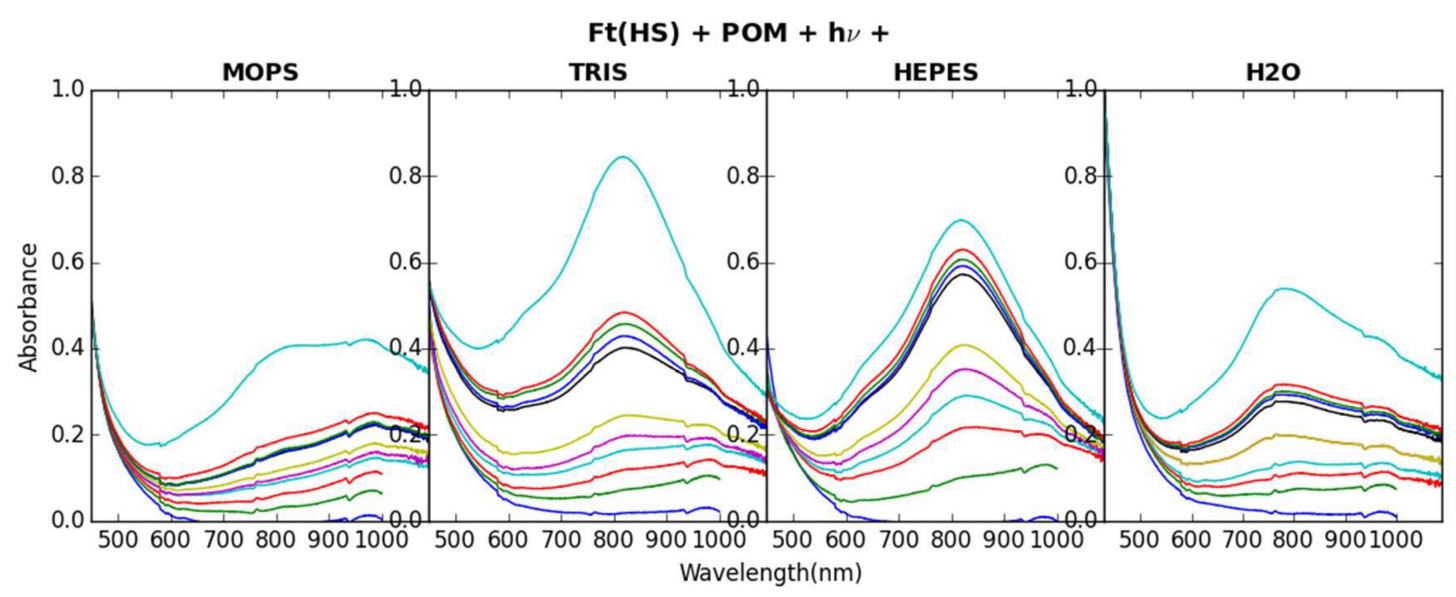

Figure S1. UV-vis spectra after irradiating the mixture of horse spleen ferritin (HSFt), citrate and POM in different media: MOPS, TRIS, HEPES and H2O.

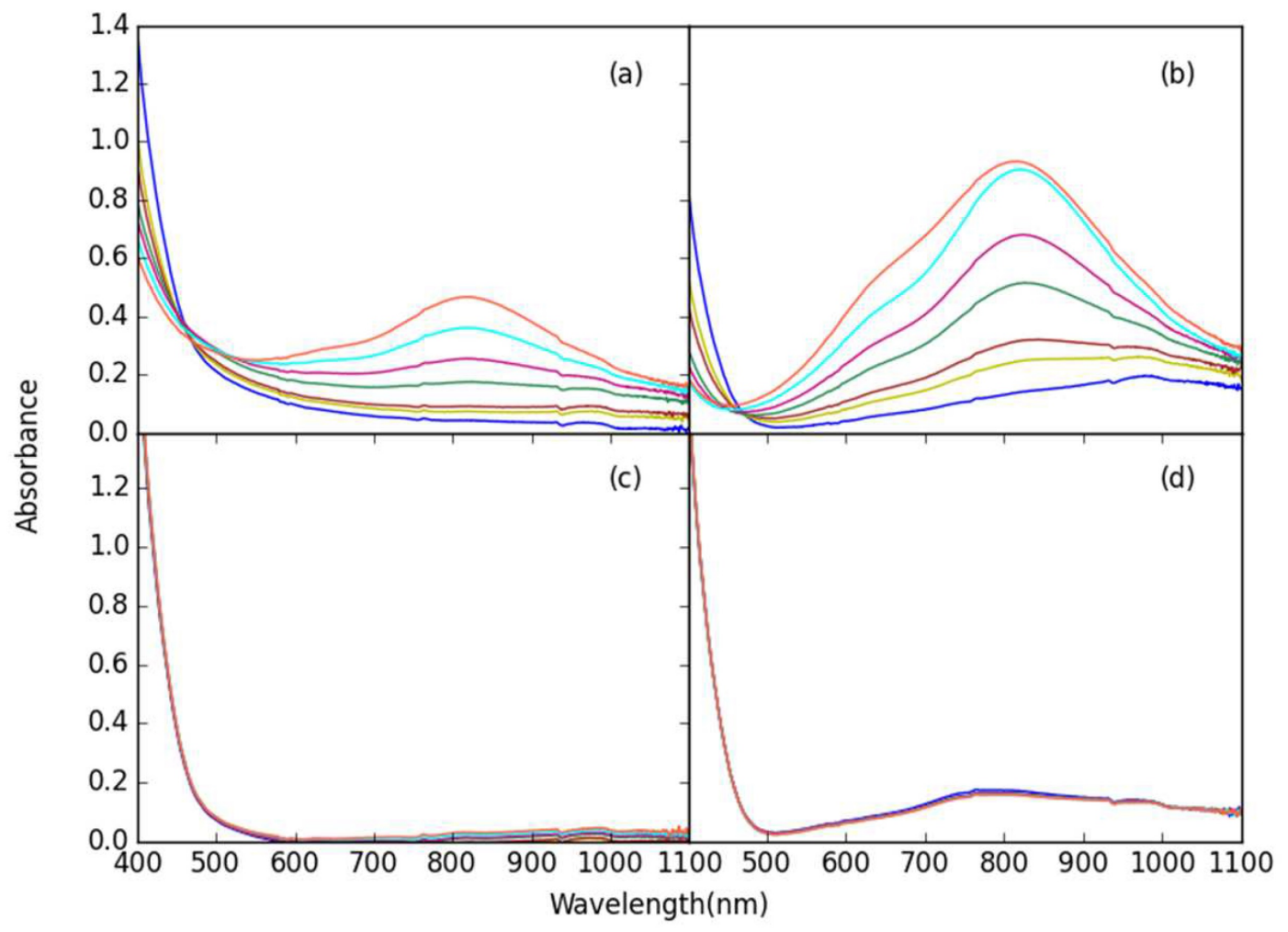

Figure S2. UV-vis spectra after irradiating (a) HSFt with POM with citrate, (b) Fe(II) 0,03uM with POM with citrate, (c) HSFt with POM without citrate and (d)Fe(II) 0,03uM with POM without citrate. 


\section{REFERENCES}

[1] Arosio, P.; Carmona, F.; Gozzelino, R.; Maccarinelli, F.; Poli, M. Biochem. J. 2015, 472, 1-15.

[2] Lane, D. J. R.; Merlot, A. M.; Huang, M. L.-H.; Bae, D.-H.; Jansson, P. J.; Sahni, S.; Kalinowski, D. S.; Richardson, D. R. Biochim. Biophys. Acta, Mol. Cell Res. 2015, 1853, 1130-1144.

[3] Honarmand Ebrahimi, K.; Hagedoorn, P.-L.; Hagen, W. R. Chem. Rev. 2015, 115, 295-326.

[4] Finazzi, D.; Arosio, P. Arch. Toxicol. 2014, 88, 1787-1802.

[5] Alkhateeb, A. A.; Connor, J. R. Biochim. Biophys. Acta, Rev. Cancer 2013, 1836, 245-254.

[6] Bou-Abdallah, F. Biochim. Biophys. Acta, Gen. Subj. 2010, 1800, 719-731.

[7] Carmona, F.; Palacios, O.; Gaĺvez, N.; Cuesta, R.; Atrian, S.; Capdevila, M.; Dominguez-Vera, J. M. Coord. Chem. Rev. 2013, 257, 2752-2764.

[8] Bradley, J. M.; Moore, G. R.; Le Brun, N. E. JBIC, J. Biol. Inorg. Chem. 2014, 19, 775-785.

[9] Chasteen, N. D.; Harrison, P. M. J. Struct. Biol. 1999, 126, 182- 194.

[10] Gaĺvez,N.; Fernańdez,B.; Sańchez,P.; Cuesta,R.; Ceolín,M.; ClementeLeoń,M.; Trasobares,S.; LoṕezHaro,M.; Calvino,J.J.; Steṕhan,O.; DominguezVera,J.M. J.Am.Chem.Soc.2008,130, 8062.

[11] Theil, E. C. Curr. Opin. Chem. Biol. 2011, 15, 304.

[12] Clemente-Leoń,M.; Coronado,E.; Soriano-Portillo,A.; Colacio, E.; Dominguez-Vera, J. M.; Gaĺvez, N.; Martín-Romero, M. T. Langmuir 2006, 22, 6993.

[13] Ensign, D.; Young, M.; Douglas, T. Inorg. Chem. 2004, 43, 3441-3446. 
[14] Kim, I.; Hosein, H.-A.; Strongin, D. R.; Douglas, T. Chem. Mater. 2002, 14, 4874-4879.

[15] Keyes, J. D.; Hilton, R. J.; Farrer, J.; Watt, R. K. J. Nanopart. Res. 2011, 13, 2563-2575.

[16] Petrucci, O. D.; Buck, D. C.; Farrer, J. K.; Watt, R. K. RSC Adv. 2014, 4, 3472-3481.

(17) Watt, R. K.; Petrucci, O. D.; Smith, T. Catal. Sci. Technol. 2013, 3, 3103.

[18] Hilton, R. J., Keyes, J. D.; Watt, R. K. Maximizing the efficiency of ferritin as a photocatalyst for applications in an artificial photosynthesis system; Proc. SPIE 7646, Nanosensors, Biosensors, and Info-Tech Sensors and Systems 2010, 76460J (March 30, 2010); DOI: 10.1117/ 12.858830.

[19] Colton, J. S.; Erickson, S. D.; Smith, T. J.; Watt, R. K. Nanotechnology 2014, 25, 135703/1-135703/7.

[20] Nikandrov,V.V.;Grä̈zel,C.K.;Moser,J.-E.;Grä̈zel,M.J. Photochem. Photobiol., B 1997, B41, 83-89.

[21] Wolszczak, M.; Gajda, J. Res. Chem. Intermed. 2010, 36, 549- 563.

[22] Paconstaninum, E. Chem. Soc. Rev. 1989, 18, 1.

[23] Zhang, T. R.; Feng, W.; Lua, R.; Bao, C. Y.; Zhang, X. T.; Li, T. J.; Zhao, Y. Y.; Yao, J. N. Mater. Chem. Phys. 2002, 78, 116.

[24] Gonzalez,A.; Gaĺvez,N.; Clemente-Leon,M.; Dominguez-Vera, J. M. Chem. Commun. 2015, 51, 10119-10122.

[25] Strandberg, R. Acta Chem. Scand. 1975, 29, 350.

[26] Santambrogio, P.; Cozzi, A.; Levi, S.; Rovida, E.; Magni, F.; Albertini, A.; Arosio, P. Protein Expression Purif. 2000, 19, 212-218. 